\title{
Research on Substation Overheating Fault On-line Monitoring Based on Support Vector Machine
}

\author{
Min $\mathrm{Xu}^{1, \mathrm{a}}$, Luona $\mathrm{Hu}^{2, \mathrm{~b}}$ and Jizhe Wang ${ }^{2, \mathrm{c}}$ \\ ${ }^{1}$ School of Electrical Engineering, Chongqing University , Chongqing 410000, China; \\ ${ }^{2}$ STATE GRID CHONGQING Information \& Telecommunication Company, Chongqing 410000 , \\ China. \\ a286272008@qq.com, b403188336@qq.com, c 269209136 @qq.com
}

Keywords: Substation, overheating fault, On-line monitoring, Support Vector Classification

\begin{abstract}
As an important part of power transition, the safe operation of substation is of great significance. In this paper, according to the characteristics of the substation equipment overheating fault and the theory of mathematical model of support vector classifier, equipment overheating fault diagnosis mechanism is established. By using inner product kernel function, the method realizes nonlinear mapping to high dimension space. The algorithm completes feature space division through the optimal hyper plane. The method is not only simple, but also has well "robust". Take substation in power plant as example, we verified the powerful function of support vector classifier and it can complete the fault diagnosis of excellent. The algorithm can completely satisfy the requirement of the traction substation equipment overheating fault on-line monitoring.
\end{abstract}

\section{Introduction}

Generally, by the time, the main substation primary equipment, primary grid and a number of secondary equipment. Primary equipment is: reactors, transformers, bus bars, surge arresters, transformers, grounding devices, etc. [3].Secondary equipment are: control switch, control cables, relays, etc.Wiring is a major bridge and a T-connection, this connection needs to be fixed by the different shapes of the clip, convergence. Every electrical equipment components are their duties to play its appropriate role in maintaining the normal operation of the equipment; which has become a main focus of substation monitoring. For the monitoring of these critical electrical equipment, not just to determine whether it can work, voltage, power, etc. are within the normal range, but also concerned about the heat and strain the situation of the equipment.Substation electrical equipment easy to heat, the damaged areas include: high voltage isolation switch, Clamps and busbars (copper, aluminum row) and so on[4].For electrical substation equipment are concerned, they play different roles among the substation.

The electrical equipment will be distributed during the normal operation of the heat, when the heat and heat emitted is balanced, the device temperature will not rise; If the device temperature continues to rise, it will have a serious impact on device performance. As the mechanical strength is lowered, the contact resistance is increased to produce a higher temperature, so that some rubber insulation material appears strain, aging and so on.Because of the current general monitoring tools can not do real-time monitoring, and therefore it can not directly read temperature data, but judging by the experience of overheating equipment failure or unstable operation of the device by indirectly reflected.This point in time be checked, they often have caused damage to the equipment.Therefore, the study of electrical substation equipment overheating fault-line monitoring system for running the substation has important practical significance.

\section{Overheating fault Factor}

Affect most intuitive troubleshooting substation overheated impact factor, atmospheric temperature.In general, the normal temperature is the actual temperature of the substation equipment, 
but the failure temperature, use of the alarm value (higher than the normal 10 degrees Celsius) over temperature.Table 1 shows the April 16, 2012, a power plant on the 1st transformer substation with a wall bushing temperature changes.

Short period of time can be approximately considered, substation hourly load is essentially the same.As can be seen from the table, the temperature of the device is significantly higher than the day and night.It indicated that the temperature of the atmospheric environment, for the equipment is a relatively large impact.Then, in the diagnosis of failure, atmospheric temperature is a negligible impact factor.But the alarm system do not take into account this factor, it set the alarm value for daytime and night are the same.Similarly, as the seasons change, the corresponding alarm values should be changed.However, if you rely on manual to change the set value, then its role is very limited.

Table 1 The temperature of wear casing wall of a phase 1 of substation transformer

\begin{tabular}{cccc}
\hline Date & Temperature $\left({ }^{\circ} \mathrm{C}\right)$ & Date & Temperature $\left({ }^{\circ} \mathrm{C}\right)$ \\
\hline 2015/4/16 0:19 & 14.1 & $2015 / 4 / 1612: 15$ & 24.4 \\
2015/4/16 1:18 & 15.2 & $2015 / 4 / 1613: 15$ & 21.1 \\
2015/4/16 2:18 & 15.8 & $2015 / 4 / 1614: 15$ & 20 \\
2015/4/16 3:18 & 13.2 & $2015 / 4 / 1615: 15$ & 20 \\
2015/4/16 4:18 & 12.6 & $2015 / 4 / 1616: 14$ & 20 \\
2015/4/16 5:17 & 12.9 & $2015 / 4 / 1617: 14$ & 19.7 \\
2015/4/16 6:17 & 12.9 & $2015 / 4 / 1618: 14$ & 18.5 \\
2015/4/16 7:17 & 13.5 & $2015 / 4 / 1619: 14$ & 16.7 \\
2015/4/16 8:17 & 16.4 & $2015 / 4 / 1620: 13$ & 16.4 \\
$2015 / 4 / 16$ 9:16 & 18.5 & $2015 / 4 / 1621: 13$ & 13.8 \\
$2015 / 4 / 1610: 16$ & 20.5 & $2015 / 4 / 1622: 13$ & 12.6 \\
$2015 / 4 / 1611: 16$ & 24.7 & $2015 / 4 / 1623: 12$ & 12.9 \\
\hline
\end{tabular}

\section{Support Vector Classifications}

Support Vector Classification (SVC), the basic idea is: by a non-linear mapping firstly, mapping from the original input vector space to a high dimensional feature space.

In this space, the uses of structural risk minimization principle to construct the optimal decision function.In this process, using the original space of the kernel function to replace the high-dimensional feature space dot product operations reduces the computational complexity.

Setting a given training set as $\left\{\left(x_{1}, y_{1}\right), \cdots,\left(x_{l}, y_{l}\right)\right\}, x_{i} \in R^{n}, y_{i} \in\{-1,1\}, l$ is the total number of samples. Nonlinear mapping from the original sample space $\varphi(\cdot), R^{n}$ mapped into a high dimensional feature space, construct optimal linear decision function $y(x)=\operatorname{sgn}[w \cdot \varphi(x)+b]$ in this high-dimensional space. Where, $\mathrm{w}$ is the weight vector; $\mathrm{b}$ is a constant.

Solving Support Vector Machine hyper plane:

$$
\left\{\begin{array}{l}
\min \frac{1}{2}\|w\|^{2} \\
\text { s.t. }\left[\left(w \cdot x_{i}\right)+b-1\right] \geq 0, i=1, \cdots, n
\end{array}\right.
$$

Lagrange function is defined as:

$$
\begin{aligned}
L(w, b, \alpha) & =\frac{1}{2}\|w\|^{2} \\
& -\sum_{i=1}^{n} \alpha_{i}\left\{y_{i}\left[\left(w \cdot x_{i}\right)+b\right]-1+\xi_{i}\right\}
\end{aligned}
$$

Derivation for $\mathrm{w}$, b: 


$$
\begin{aligned}
& \left\{\begin{array}{l}
\frac{\partial L}{\partial w}=0 \Rightarrow w=\sum_{i=1}^{n} \alpha_{i} y_{i} x_{i} \\
\frac{\partial L}{\partial b}=0 \Rightarrow \sum_{i=1}^{n} \alpha_{i} y_{i}=0
\end{array}\right. \\
& L(w, b, \alpha)=\sum_{i=1}^{n} \alpha_{i}-\frac{1}{2} \sum_{i, j=1}^{n} y_{i} y_{j} \alpha_{i} \alpha_{j}\left(x_{i} \cdot x_{j}\right)
\end{aligned}
$$

To (3) into (2): The constraint is:

$$
\sum_{i=1}^{n} y_{i} \alpha_{i}=0 \quad \alpha_{i}>0, i=1,2, \cdots, n
$$

Introducing a non-negative slack variables, and Penalty parameter $\mathrm{C}($ When $\mathrm{C}$ infinity, Is the original classification hyper plane),that is C-SVC.

Use Lagrange function, You can get the dual problem of the problem:

$$
\max L_{D}=\sum_{i} \alpha_{i}-\frac{1}{2} \sum_{i+j} \alpha_{i} \alpha_{j} y_{i} y_{j} \varphi\left(x_{i}\right) \varphi\left(x_{j}\right) \quad\left\{\begin{array}{l}
\alpha^{T} y=0 \\
0 \leq \alpha \leq C
\end{array}\right.
$$

Setting kernel $K\left(x_{i}, x_{j}\right)=\varphi\left(x_{i}\right) \cdot \varphi\left(x_{j}\right)$, then

$$
\Omega_{k l}=y_{k} y_{l} K\left(x_{k}, x_{l}\right)
$$

Get the output results:

$$
\begin{aligned}
& \quad y(x)=\operatorname{sgn}\left[\sum_{k=1}^{N} \alpha_{k} y_{k} K\left(x, x_{k}\right)+b\right] \\
& \alpha_{k} \in R^{N} 。
\end{aligned}
$$

\section{Case Study}

In order to verify the effectiveness of the proposed method, we use the MATLAB platform, the proposed classification mechanism to analyze an example.Experimental selected RBF kernel function as kernel function to complete the original data to the high-dimensional space nonlinear mapping.The experimental data collected in the substation in a power plant April 16, 2015.

The most direct impact factor for atmospheric temperature and the device temperature is overheating fault . so each input sample is two-dimensional in the experiment.The final algorithm returns the value by 1 or -1 components.If the sample which judged to be the device temperature is normal, the return value is 1 ; if the sample is judged to be overheating, the return value is -1 .

In the experiment, the training set is composed of 50 samples and 50 normal temperature failure temperature samples, validation set from three samples of the normal temperature and the temperature of three samples fault composition.

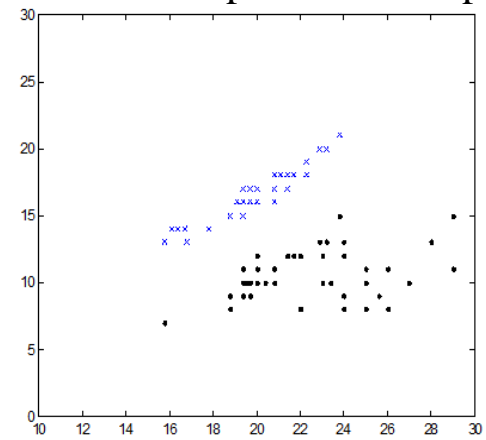

Fig. 1 The training dataset

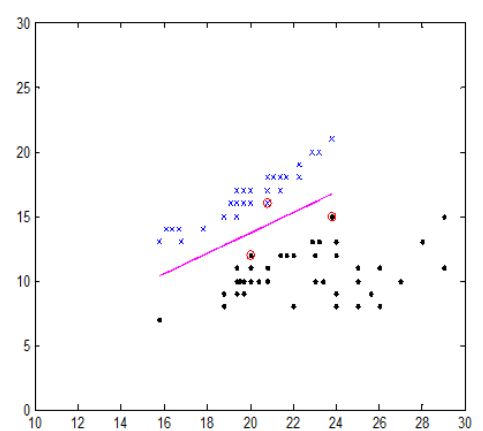

Fig.2 The support vector and classification faces

As shown in Figure 1. X-axis is the temperature of the device. Y-axis is the air temperature. Blue $\mathrm{X}$-type icon is the normal temperature of the sample values, the black dot is the sample failure temperature values. 
The training set of sample input to the C-SVC, to establish the initial model and training, you can get support vector classification and optimal surface. Shown in Figure 2, the red circle of support vector, the red line is the optimal classification surface.

Finally, enter the validation set of samples C-SVC, get the classification results, you can verify the validity of the algorithm. As shown in Figure 3.

Blue five-pointed star is the validation sample data set. As can be seen from the figure, the three centralized validation samples and three normal temperature failure temperature samples are correctly sorting process. Thus, it can be concluded that the proposed algorithm powerful classification mechanism, able to complete the outstanding overheating fault diagnosis.

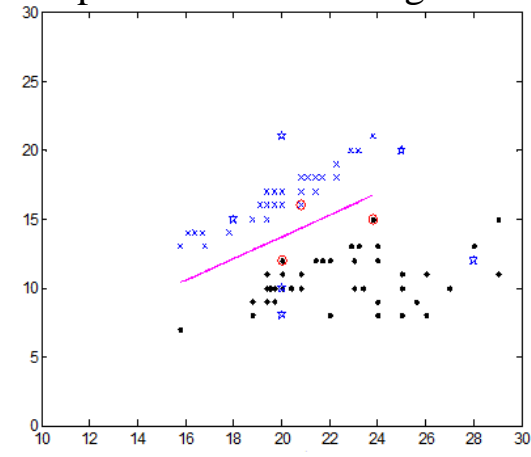

Fig.3 The classification results

\section{Conclusion}

According to the characteristics of substation equipment overheating fault, we use SVM classifier mathematical theoretical model, establish to determine the failure mechanism of the equipment from overheating.

The methods utilizing product kernel function to achieve the high-dimensional space nonlinear mapping, and through optimal hyperplane in the feature space to be divided, not only simple algorithm, but also has better robustness.In a power plant substation, for example, to verify the SVM classifier powerful, able to complete the outstanding fault diagnosis, fully meet the substation equipment overheating fault line monitoring requirements.

\section{Reference}

[1]PD Kayano, MS Silva, LC Magrini. Distribution substation transformer and circuit breaker diagnoses with the assistance of real-time monitoring[C]. IEEE Transmission \& Distribution Conference \& Exposition, 2004.

[2]Federico Sforza, Vittorio Lippi. Support vector machine classification on a biased training set : Multi-jet background rejection at hadron colliders. Nuclear Instruments and Methods in Physics Research A. 2013(722), 11-19.

[3]Reza Arghandeh, Ahmet Onenb, Jaesung Jungb, Robert P. Broadwater. Harmonic interactions of multiple distributed energy resources in power distribution networks. Electric Power Systems Research.Volume 105, December 2013, Pages 124-133.

[4]Manjur Ahmeda, Hudab, Nor Ashidi Mat Isaa, Recursive construction of output-context fuzzy systems for the condition monitoring of electrical hotspots based on infrared thermography. Engineering Applications of Artificial Intelligence.Volume 39, March 2015, Pages 120-131. 